\title{
Das übersehene Medium: Zur Leistung und Bedeutung von Zeitschriften für eine sich ausdifferenzierende Gesellschaft. Eine Analyse am Beispiel des Schweizer Zeitschriftenmarktes
}

\author{
Jarren, Otfried ; Oehmer, Franziska ; Dioh, Yuvviki
}

\begin{abstract}
In segmentär differenzierten, individualisierten pluralen Gesellschaften sind Individuen wie soziale Gruppen zunehmend auf medial vermittelte spezifische Repräsentations-, Informations- wie Kommunikationsmöglichkeiten angewiesen. Diese Leistung erbringen spezialisierte publizistische Medien. Die universellen, tagesaktuellen publizistischen (Massen-)Medien, deren Funktion in der gesamtgesellschaftlichen Reflexion und Realitätskonstruktion liegt, leisten dies funktional nicht. Für die zielgerichtete Informationssuche wird vor allem auf Zeitschriften als thematisch spezialisierte wie sozial auf Gruppen und Interesse gerichtete Medien - und neuerdings auch auf Onlineangebote wie Social Media - zurückgegriffen. Den kommunikativen Leistungen von Zeitschriften im Sinne der Repräsentation von sozialen Interessen wie des Austausches spezifischer Informationen wurde in der Kommunikationswissenschaft wenig Beachtung geschenkt. Der Beitrag beabsichtigt, diese Forschungslücke zu schließen. Gefragt wird nach der Bedeutung von Zeitschriften für gesellschaftliche Differenzierungsprozesse. Dahinter liegt die Annahme, dass sich die Ausdifferenzierung und Pluralisierung in modernen, pluralen Gesellschaften von den Themen, Absendern und Zielstellungen des Zeitschriftenangebots ablesen lässt. Diese Grundthese beruht auf differenzierungstheoretischen und mediensystemischen Überlegungen, welche im Rahmen einer diachronen Strukturanalyse des Schweizer Zeitschriftenmarkts (1975-2016) überprüft werden sollen.
\end{abstract}

DOI: https://doi.org/10.24989/medienjournal.v43i3.1833

Posted at the Zurich Open Repository and Archive, University of Zurich

ZORA URL: https://doi.org/10.5167/uzh-184737

Journal Article

Published Version

Originally published at:

Jarren, Otfried; Oehmer, Franziska; Dioh, Yuvviki (2020). Das übersehene Medium: Zur Leistung und Bedeutung von Zeitschriften für eine sich ausdifferenzierende Gesellschaft. Eine Analyse am Beispiel des Schweizer Zeitschriftenmarktes. Medien Journal, 43(3):46-68.

DOI: https://doi.org/10.24989/medienjournal.v43i3.1833 


\title{
Das übersehene Medium \\ Zur Leistung und Bedeutung von Zeitschriften für eine sich ausdifferenzierende Gesellschaft. Eine Analyse am Beispiel des \\ Schweizer Zeitschriftenmarktes"
}

\begin{abstract}
In segmentär differenzierten, individualisierten pluralen Gesellschaften sind Individuen wie soziale Gruppen zunehmend auf medial vermittelte spezifische Repräsentations-, Informations- wie Kommunikationsmöglichkeiten angewiesen. Diese Leistung erbringen spezialisierte publizistische Medien. Die universellen, tagesaktuellen publizistischen (Massen-)Medien, deren Funktion in der gesamtgesellschaftlichen Reflexion und Realitätskonstruktion liegt, leisten dies funktional nicht. Für die zielgerichtete Informationssuche wird vor allem auf Zeitschriften als thematisch spezialisierte wie sozial auf Gruppen und Interesse gerichtete Medien - und neuerdings auch auf Onlineangebote wie Social Media - zurückgegriffen. Den kommunikativen Leistungen von Zeitschriften im Sinne der Repräsentation von sozialen Interessen wie des Austausches spezifischer Informationen wurde in der Kommunikationswissenschaft wenig Beachtung geschenkt. Der Beitrag beabsichtigt, diese Forschungslücke zu schließen. Gefragt wird nach der Bedeutung von Zeitschriften für gesellschaftliche Differenzierungsprozesse. Dahinter liegt die Annahme, dass sich die Ausdifferenzierung und Pluralisierung in modernen, pluralen Gesellschaften von den Themen, Absendern und Zielstellungen des Zeitschriftenangebots ablesen lässt. Diese Grundthese beruht auf differenzierungstheoretischen und mediensystemischen Überlegungen, welche im Rahmen einer diachronen Strukturanalyse des Schweizer Zeitschriftenmarkts (1975-2016) überprüft werden sollen.
\end{abstract}

Keywords: Zeitschriften, Schweizer Zeitschriftenmarkt, Differenzierung, Mediensystem, Strukturanalyse

\section{Einleitung}

In modernen, segmentär ausdifferenzierten und hochgradig individualisierten, pluralen Gesellschaften sind Individuen und soziale Gruppen in zunehmendem Maße auf spezialisierte publizistischen Medien angewiesen (Wessler, 2002): Diese Medien re-

1) Das Projekt wurde durch den Schweizerischen Nationalfonds gefördert. Unser Dank gilt insbesondere Prof. Dr. Olaf Jandura für seine sehr wertvollen Anregungen und das konstruktive Feedback zum Manuskript. Das Autorenteam dankt zudem Prof. Dr. Andreas Vogel, Institut für Presseforschung und Publikumsanalysen, für die kritische Begleitung des Projekts. 
präsentieren die sozialen Interessen, ermöglichen Binnenkommunikation für spezialisierte soziale Gruppen und stellen spezifische Informationen bereit. Vor allem um Entscheidungsoptionen für eigenes Handeln abklären, bewerten und legitimieren zu können, benötigen Akteure (fach)spezifische und thematisch spezialisierte Kenntnisse und Wissen über mögliche Handlungsalternativen und -konsequenzen. Und sie benötigen den kommunikativen Austausch mit anderen, um ihre Handlungs- wie Entscheidungsoptionen spiegeln zu können (Kübler, 2009; Schimank, 2005; Stehr \& Adolf, 2015). Diese werden ihnen nicht von den aktuellen, universellen publizistischen Massenmedien, deren Funktion vor allem in der gesamtgesellschaftlichen Reflexion und Realitätskonstruktion (Hasebrink \& Domeyer, 2010, S. 52) liegt, zur Verfügung gestellt, sondern von den thematisch wie sozial spezialisierten publizistischen Medien. Neben Onlinemedien und Social Media-Angeboten, die diese Leistung seit jüngerer Zeit erbringen, wird dazu zielgerichtet auf Zeitschriften zurückgegriffen (IVW-Auflagenstatistik, 2017; Jentges, Brändli, Donges \& Jarren, 2013; Strassner, 1997, S.26ff; Vogel, 2002, S.12). Das Marktvolumen der Zeitschriften ist, trotz der Medien- bzw. Pressekrise, relativ stabil geblieben ('Tschörtner \& Schenk, 2009; Vogel, 2010, 2014). Mehr als nur das: Neuerscheinungen der letzten zehn Jahre erreichten in der Summe höhere Reichweiten, die Lesernutzung blieb stabil, der Auflagenverkauf ging nur leicht zurück, und der Abonnementverkauf blieb sogar stabil (IVW-Auflagestatistik, 2017; Verband Deutscher Zeitschriftenverleger, 2018).

Wie von relevanten ZeitschriftenforscherInnen mehrfach und seit den Anfängen der Zeitschriftenforschung betont (Bohrmann \& Schneider, 1975; Bohrmann, 2002; Gerlach, 1988; Holmes, 2007; Stöber, 2002; Vogel, 2002; Vogel \& Holtz-Bacha, 2002) wird jedoch der anhaltenden Bedeutung von Zeitschriften als einem Phänomen gesellschaftlicher und medialer Differenzierung in der kommunikationswissenschaftlichen Forschung kaum Rechnung getragen: Bei der Deskription, Analyse und dem Vergleich von (nationalen) Mediensystemen und deren gesellschaftlichen Funktionen wird der Fokus vor allem auf die aktuellen und universellen publizistischen Massenmedien (Tagespresse, Radio- und TV) gelegt und dabei häufig auf sogenannte Leitmedien (politische Tages- wie Wochenpresse) eingeschränkt. Dies deshalb, weil diese Medien einen erwartbaren Beitrag zur politischen Kommunikation leisten, indem sie besonders relevante Prozesse der Interessenartikulation, -aggregation wie auch -durchsetzung darstellen und kommentieren (Donges \& Jarren, 2017). In der politischen Kommunikationsforschung stehen ebenso die sogenannten Leit- oder Qualitätsmedien im Fokus. Dabei wird übersehen, dass Zeitschriften wie andere Fachmedien zur gesellschaftlichen Interessenartikulation, zur Debatte von Positionen wie zur Verbreitung von Wissen, auch mit Bezug zu politischen Vorgängen, relevant sind. Aber auch bei wissenschaftlichen Auseinandersetzungen mit Zeitschriften stand nicht der funktionale Leistungsbeitrag dieser Mediengattung für moderne differenzierte Gesellschaften im Vordergrund: Vor allem galt das Erkenntnisinteresse entweder der ökonomischen Leistungsfähigkeit von Zeitschriften im Rahmen von Marktanalysen (Heinrich, 2002; 
Nowak, 2009; Seufert, 2004; Tschörtner \& Schenk, 2009; Vogel 2010; Vogel 2014) oder den spezifischen Inhalten (bspw. dem Frauenbild in Zeitschriften: Beetham 1996; Davalos, D.B., Davalos, R.A. \& Layton, 2016; Hermes, 2010; Sakamoto, 1999; Schlenker, Caron \& Haltemann, 1998; Stephenson, 2007; Ytre-Arne, 2011, oder Gesundheitsinformationen: Aubrey \& Hahn, 2016; Bazzini, Pepper, Swofford \& Cochran, 2015; Hinnant, 2009; Mathews, Laditka, S.B., Laditka, J.N. \& Friedman, 2009; Ringelmann, 1991; Wilson, Smith, Peel, Robertson \& Kypri, 2017). Zudem herrschen Fallstudien vor (Cauers, 2009; Fiedler, 2003; Schmolke, 2002; Rössler \& Ott, 2002; Röttger, 2002).

Der vorliegende Beitrag will diese Forschungslücke schließen. Gefragt wird nach der Rolle von Zeitschriften für gesellschaftliche Differenzierungsprozesse. Die Grundthese ist, dass sich die Ausdifferenzierung und Pluralisierung sozialer Gruppen in modernen, pluralen Gesellschaften vor allem auch aufgrund ökonomischer Erwägungen in den Themen, Absendern und Zielstellungen des Zeitschriftenangebots widerspiegelt. Zunächst sollen dazu differenzierungstheoretische Überlegungen auch mit Blick auf das Mediensystem vorgestellt, diskutiert und zur Thesenbildung nutzbar gemacht werden. Im Anschluss folgt die Darstellung der gewählten Methode und des Designs. Die Überprüfung der Thesen erfolgt im darauffolgenden Ergebniskapitel, das abschließend diskutiert wird.

\section{Differenzierung der Gesellschaft}

Moderne Gesellschaften sind vielfältig ausdifferenzierte Gesellschaften. $\mathrm{Zu}$ den besonderen Merkmalen der modernen Gesellschaft gehören Individualisierung, ein hohes Maß an sozialer wie räumlicher Mobilität, die Pluralisierung der Wertvorstellung, diverse Lebensstile wie -weisen (Hradil, 2002; Inglehart, 2018). In der Forschung werden vier Differenzierungsformen unterschieden: die segmentäre Differenzierung (Aufteilung in Familie, Gruppe, Nation, etc.), die Differenzierung nach Zentrum und Peripherie (wie Stadt und Land), die stratifikatorische Differenzierung (Schichten) sowie die funktionale Differenzierung (Teilsysteme) (Luhmann, 1997, S. 613; Schimank, 1996, S. 151-152). In der gesellschaftlichen Evolution hat sich der Primat der Differenzierungsformen verschoben: von der segmentären zur heute dominanten funktionalen Differenzierung. Dabei verlaufen die Differenzierungsprozesse aber nicht linear und es existieren vielfältige Interdependenzen zwischen ihnen. So hat die funktionale Differenzierung, die die Bildung sozialer Funktionssysteme zur Folge hat, durch Rationalisierung und Effizienzsteigerungen u. a. im ökonomischen System eine die Arbeitsbedingungen verändernde und zudem wohlstandsfördernde Wirkung entfaltet, so dass mehr Zeit für andere Dinge als Arbeit möglich wurde (Hradil, 2002). Die freie Zeit wird für vielfältige Freizeitaktivitäten im wie außerhalb des Hauses genutzt und hat auch Auswirkungen auf das Interesse an spezialisierten Medienangeboten, so um sich über Freizeitgestaltung, Sport u. a. m. zu informieren. Steigendes Haushaltseinkommen und mehr Freizeit haben auch den Medienkonsum, zumal bei den spe- 
zialisierten Medien, beeinflusst (Kübler, 2009, S. 41ff). Im Freizeitbereich haben sich spezialisierte Verbände ebenso etabliert wie weitere soziale Gruppen, die sich auch über spezialisierte Medien konstituieren (Kuhn, 2018, S.299). Spezialisierte Organisationen wie Kunden bedienen sich im Wettbewerb spezialisierter Medien, um ihre jeweiligen Zielgruppen zu erreichen. Sozialen Gruppen dienen diese Medien zur Binnenkommunikation, zur Orientierung - so über Waren und Dienstleistungen - wie zur spezifischen Information (Babic \& Jandura, 2017; Kuhn 2018; Lüdtke, 2000).

Der sich beschleunigende gesellschaftliche Wertewandel, der zur Individualisierung, zur Pluralisierung und zur Wertevielfalt beitrug, hat die Effekte segmentärer Differenzierung wieder deutlicher werden lassen: Die Gesellschaft besteht aus vielen sozialen Gruppen, aus verschiedenen (Interessen-)Gemeinschaften, aus verschiedenen sozialen Milieus (Reckwitz, 2018). Diese werden durch spezialisierte Medien wie Medienangebote sichtbar und repräsentiert. Zwei zentrale Wirkmechanismen werden dabei als ursächlich für die Repräsentation einer ausdifferenzierten Gesellschaft in einem ausdifferenzierten Zeitschriftenangebot vermutet: Erstens versuchen kommerzielle Verlage vor allem aus ökonomischem Kalkül die neu gebildeten und organisierten $\mathrm{Pu}$ blikumsinteressen und -bedürfnisse mit einem entsprechenden Medienangebot zu befriedigen. Zweitens finden die gebildeten Interessen auch häufig Ausdruck in neuen Organisationsformen wie Verbänden oder Vereinen, die wiederum selbstständig Zeitschriften für binnenkommunikative Austausch- und Bindungsprozesse ihrer Mitglieder und Angehörigen über spezifische Themen herausgeben. Die jeweiligen spezialisierten publizistischen Medien der Gesellschaft korrespondieren also mit den Differenzierungsformen und den sich daraus ergebenden sozialen Anforderungen.

\subsection{Differenziertes publizistisches Mediensystem}

Im Zuge der funktionalen Differenzierung nehmen die universell und aktuell orientierten Massenmedien der Gesellschaft die Funktion der Selbstbeobachtung ein: Sie greifen Themen aus allen gesellschaftlichen Teilsystemen (Wirtschaft, Sport oder Kultur etc.) auf (Blöbaum, 1994, 2000). Dabei fokussieren sie insbesondere auf solche Themen, die an das politische System adressiert sind, weil die Politik für die Herstellung allgemeinverbindlicher Entscheidungen zuständig ist. Diese Thematisierung ist für alle Akteure von besonderer Relevanz - funktional wie normativ (Luhmann, 2009). Die universellen, aktuellen Massenmedien tragen zur kollektiven Interessenfindung, -artikulation, -aushandlung wie zur Interessenrepräsentation bei. Sie tun dies, indem sie sich auf die korporativen und kollektiven Akteure der Gesellschaft beziehen im Hinblick auf die Herstellung allgemeinverbindlicher (politischer) Entscheidungen. Diese sollen die einzelnen Bürgerinnen und Bürger kennen, um sich politisch zu betätigen oder den politischen Institutionen für die Entscheidungen die Zustimmung geben zu können (so in Abstimmungen oder Wahlen). Durch die Leistungserbringung der allgemeinen, universellen Medien sollen die Bürgerinnen und Bürger am gesellschaftlichen, vor allem am politischen Geschehen, teilhaben (Donges \& Jarren, 2017). Eine 
andere Leistung erbringen die nicht-aktuellen (die spezialisierten) Medien, die sich in Form von Special Interest-, Very Special Interest-, Fach- und Peer-Medien institutionalisiert haben (vgl. dazu den Beitrag von Donges \& Jarren in diesem Heft). Sie sind das Produkt sowie ein Ausdruck der segmentär differenzierten Gesellschaft. Die spezialisierten publizistischen Medien dienen den Akteuren zur Findung, Aushandlung, Formulierung wie Anmeldung von sozialen, kulturellen, ökonomischen und politischen Interessen (Wessler, 2002). Sie werden zudem für das Treffen individueller (lebensweltlicher) Entscheidungen genutzt.

Massenmedien wie spezialisierte Medien sind also höchst unterschiedlich institutionalisiert, organisiert und publizistisch ausgerichtet. Sie bilden zusammen das publizistische Mediensystem, wobei die universellen, aktuellen Medien vorrangig der funktionalen Differenzierung und die spezialisierten publizistischen Medien vor allem der segmentär differenzierten Gesellschaft entsprechen (Blöbaum, 1994): Durch Selbstbeobachtung (Leistung der Massenmedien) und Selbstorganisation (Leistung der spezialisierten Medien) erfolgt über das gesamte Mediensystem eine Form der „Multiinklusion" (Nassehi, 2004, S. 111), so in dem durch die beteiligten Medienorganisationen in unterschiedlicher Weise die Ansprache, Teilhabe wie Teilnahme des Publikums, der Ermöglichung von binnen- wie außenkommunikativen Austauschformen, die Bereitstellung von Informationen wie Wissen u. a. m. erfolgt. Die Formen der Inklusion bei den Medienorganisationen variieren entsprechend der jeweiligen Zielsetzungen wie den Inklusionsbedürfnissen der Leistungsabnehmer. Bei den universellen Medien sind die Inklusionsformen sehr formal und wenig auf Kollaboration, sondern mehr auf Repräsentation von hochgradig verdichteten Interessen (der kollektiven und korporativen Akteure) angelegt. Die spezialisierten Medien hingegen bieten Formen der Kollaboration von Akteuren wie Gruppen, so auch bspw. bei der Autorenschaft, an (Dernbach, 2010).

Grundsätzlich sind dabei alle publizistischen Medien jedem Gesellschaftsmitglied zugänglich. Die Individuen sind damit faktisch stets durch multiple Partialinklusionen normativ, kognitiv, sozial und evaluativ in das Mediensystem - und darüber vermittelt in die Funktionssysteme der Gesellschaft wie auch in die Sozialsysteme der Lebenswelt - inkludiert. Diese Inklusion erfolgt in der Lebenswelt dabei dominant durch Leistungsbeziehungen zwischen Einzelnen und Organisationen, die durch diese kommunikativ vermittelt sind. Und vor allem die (spezialisierten) Medien sind für die individuelle Selbstorganisation relevant, weil sie eine allgemeine, kommunikative Teilhabe ohne stark formalisierte Regeln (wie Rollen) ermöglichen (vgl. Donges \& Jarren in diesem Heft).

Die universellen, aktuellen Medien konstituieren und repräsentieren also die funktional differenzierte Gesellschaft, wie sich auch an der Spartenstruktur journalistischer Angebote und der Ressortstruktur von Redaktionen ablesen lässt. Zum einen ermöglicht der Journalismus mit seiner Berichterstattung und Kommentierung intersystemisch die wechselseitige Beobachtung, Beeinflussung und Verhandlung zwischen Teil- 
systemen, wofür er Ereignisse mit „Mehrsystemrelevanz“ zum Anlass nimmt (Kohring, 2005, S. 255-278). Zum anderen koordiniert er intrasystemisch das Verhältnis zwischen den Akteuren in Leistungs- und Publikumsrollen, z.B. zwischen Politikern und Bürgern, Unternehmern und Kunden, Medizinern und Patienten usw. (Neuberger, 2016, S. 351-362), die sich zu Interessengruppen formieren. Der Journalismus orientiert sich am Inklusionsprofil seines Publikums, das an zahlreichen Teilsystemen partizipiert (Burzan et al., 2008, S. 28; Schimank, 2001).

Mit dem Konzept der spezialisierten Medien wird auf die Folgen sowohl der funktionalen (Ausbildung von spezialisierten Sozialsystemen in sozialen Teilsystemen) als auch - und vor allem - der segmentären Differenzierung (Bildung von spezifischen Interessen wie Gruppen) Bezug genommen. Die moderne Gegenwartsgesellschaft wird nämlich immer stärker durch diese Formen der Differenzierung geprägt (Kern, 2011; Nassehi, 2004; Schimank, 2005; Spellerberg, 1996), weil Einzelne, Gruppen wie Netzwerke im Zuge des Differenzierungsprozesses mehr und mehr ihre Interessen entdecken und zur Geltung bringen: Freisetzung aus Traditionsbindungen, Enttraditionalisierung der Lebensführung, Zunahme an räumlicher wie sozialer Mobilität, gewandelte Interessen und neue Formen der Interessenorganisation, neue Arbeits- und folglich auch Freizeitstrukturen (Zeitstrukturen), Zunahme des Selbstgestaltungsanspruches, Geltendmachung unterschiedlicher kultureller Normen wie Werte u.a.m. führen zur Individualisierung und Pluralisierung auf der Mikro-Ebene der Gesellschaft. Peters (1993) bezeichnet diesen Prozess als plurale Differenzierung.

\subsection{Thesen der Analyse des Zeitschriftenmarktes}

Aufbauend auf den differenzierungstheoretischen Ausführungen zu gesellschaftlichen und medialen Entwicklungen lässt sich mit Blick auf die thematische Vielfalt der publizierten Zeitschriftentitel folgende Annahme formulieren:

These 1: Themenbereiche von Zeitschriften

Es kann angenommen werden, dass sich in modernen, pluralen Gesellschaften die Ausdifferenzierung und Pluralisierung sozialer Gruppen im Zeitschriftenangebot themenspezifisch widerspiegelt.

Die spezialisierten Medien konstituieren vor allem für die Individuen, Gruppen wie Netzwerke konkrete wie spezielle Kommunikationszusammenhänge, mittels derer sie einerseits ihre Interessen finden, entwickeln und austauschen und mit denen sie andererseits für Organisationen als potentielle Leistungsempfänger (Konsum, Mitgliedschaft etc.) erreichbar und ansprechbar werden (Kuhn, 2018; Straßner, 1997). So geben Organisationen (wie bspw. Verbände, Parteien, Unternehmen u.a.m.) - neben Verlagen - selbst Publikationen heraus, bspw. als Corporate Publishing, und treten damit als neue Akteure im Zeitschriftensektor in Erscheinung. Spezialisierte Verlage agieren als strukturierende Akteure, indem sie die Interessen von potenziellen Publika mit den Interessen von Marktorganisationen koppeln. Im Zuge dieser Entwicklungen entsteht 
auch der Fachjournalismus als Intermediär zwischen spezialisiertem Publikum und verantwortlichen Entscheidungsträgern (Dernbach, 2010).

These 2: Absender von Zeitschriften

Diesen Überlegungen folgend kann angenommen werden, dass sich im Zuge gesellschaftlicher Differenzierungsprozesse des Mediensystems im Zeitverlauf vermehrt verschiedene Absender (Verlage, Unternehmen, Vereine, Verbände etc.), die Zeitschriften publizieren bzw. herausgeben, nachweisen lassen.

Vor allem die spezialisierten Medien dienen, über den unmittelbaren ökonomischen (Zeitschriften-Verlag) oder interessenpolitischen (Verband) Zweck der beteiligten Organisationen hinaus, der Darstellung, Diskussion wie Reflexion lebensweltlicher Anforderungen von Einzelnen und Gruppen. Neben kommerziellen Verlagen geben auch zunehmend Verbände oder Vereine Zeitschriften heraus, mit denen sie eigene thematische und auch organisatorische Interessen, wie bspw. die Werbung und Bindung von (neuen) Mitgliedern, verfolgen. Zusätzlich können mit der Publikation und Herausgabe einer Zeitschrift auch ökonomische Zwecke verfolgt werden.

These 3: Zweck

Es erscheint daher plausibel anzunehmen, dass sich die Publikationszwecke von Zeitschriften im Zeitverlauf den Bedürfnissen der ausdifferenzierten Gesellschaft anpassen: $\mathrm{Zu}$ nehmend lassen sich neben rein ökonomischen Zielstellungen auch Zwecke wie Binnenkommunikation zwischen Mitgliedern oder zur Kundenbindung feststellen.

Zeitschriften tragen damit zum Austausch von Informationen, zur individuellen Interessenfindung wie -definition und dadurch zur Selbstorganisation der Gesellschaft bei. Sie sind aber auch für Konsumentscheidungen relevant. Spezialisierte Medien ermöglichen und repräsentieren die segmentär differenzierte Gesellschaft. Interessenfindung wie -anmeldung erfordern nämlich anhaltende (binnen-)kommunikative Verständigungsprozesse, die nicht von aktuellen, universellen Medien funktional geleistet werden (können) (Kuhn, 2018).

In diesen Prozessen, in denen Wissen in Form vernetzter Informationen entsteht, sind alle Formen von Zeitschriften wie aber auch spezialisierte Radio- und Fernsehprogramme und neu ebenso Social Media relevant. Zeitschriften können als Ausdruck, Katalysator und Beschleuniger der segmentären Differenzierung verstanden werden. Sie ermöglichen Einzelnen, Netzwerken wie Gruppen die (Selbst-)Präsentation, den Wissensaustausch, die Interessenaushandlung wie die (Selbst-)Organisation. Sie sind somit auch für das Treffen individueller Entscheidung von Bedeutung.

Die hochgradige Individualisierung und Pluralisierung von modernen Gesellschaften lässt sich auf den Wertewandel, welcher die Veränderung von spezifischen gesellschaftlichen und individuellen Werten und Wertorientierungen über einen bestimmten Zeitraum beschreibt, zurückführen (Hradil, 2002; Inglehart, 2018). Grundsätzlich wird bezüglich des Wertewandels in (ehemaligen) Industriegesellschaften vom Rückgang traditioneller, materialistischer und der Zunahme progressiver, postmoderner oder postmaterialistischer Werte und Wertorientierungen gesprochen (Bürklin, 
1988; Hillmann, 2001; Inglehart, 2018). Im Rahmen von Wertewandel-Forschungsprojekten wie z.B. der World Value Survey, der European Value Study, dem International Social Survey Programme (ISSP), welche größtenteils auf dem Inglehart-WerteIndex basieren, werden u.a. die Abnahme von strikten Arbeits- und Berufswerten (Disziplin, Ordnung, Fleiss etc.) sowie eine Zunahme und stärkere Gewichtung von hedonistischen und individualistischen Bedürfnissen (Selbstentfaltung, Emanzipation, Spannung etc.) beobachtet (Franzen \& Vogl, 2013; Hug \& Kriesi, 2010; Inglehart, 2018). Grund für diese Wertverschiebung ist das nachkriegszeitliche Wirtschaftswachstum und die anhaltende Wohlstandszunahme in westlichen Gesellschaften. Träger dieser postmaterialistischen Wertprioritäten waren die nachfolgenden Generationen, welche immer mehr in Zusammenhang mit anhaltend verbessernden Lebensbedingungen aufwuchsen (Bürklin, 1988; Hillmann, 2001; Voll, 2001). Aufgrund der Abkehr von traditionellen Werteorientierungen, zugleich verbunden mit der Bildung (neuer) spezifischer sozialer Gruppen, entstehen verschiedenste soziale Milieus und Szenen gemäß gruppenspezifischen Existenzformen mit erhöhter Binnenkommunikation, spezifischen Deutungsmustern, Geschmackskulturen und Lebensphilosophien (Kübler, 2009; Schulze, 2005). Die Produktion hat sich vom Primat des primären Sektors hin zum Primat des sekundären Sektors sowie der Technisierung von Produktion wie Hausarbeit gewandelt. Das hat zu einem Anstieg der frei verfügbaren Zeit in der Bevölkerung geführt. Das Vorhandensein einer solchen Zeit ermöglicht hedonistische Wertorientierungen. Der Zuwachs an Freizeit ermöglicht vielfältige Formen der Interessenrealisation, das sich in unterschiedlichen Lebensstilen ausdrückt (Lüdtke, 2000).

These 4: Themen Zeitschrift, Fokus Freizeit

Es kann daher angenommen werden, dass dieses ausgeprägtere, differenziertere Freizeitverhalten zu einem Zuwachs an Zeitschriften in diesem Themenbereich führt, da der Zeitschriftenmarkt als Teil des Erlebnismarkts angesehen werden kann.

Die gesellschaftliche Differenzierung, Individualisierung und Pluralisierung ist nicht nur hinsichtlich des Freizeitverhaltens beobachtbar, sondern auch bei näherer Betrachtung des berufsstrukturellen Wandels der letzten 40 Jahre. Es besteht der Konsens, dass gesamtgesellschaftliche Entwicklungen wie die Modernisierung einen starken Einfluss auf Berufsstrukturen ausüben bzw. ausgeübt haben (Berger, 1986; Schwahn, Mai \& Braig, 2018; Sheldon, 2005; Zangger, Glauser \& Becker, 2018). Nachkriegsgesellschaftliche Symptome wie die Grenzen des (wirtschaftlichen) Wachstums, die Endlichkeit der natürlichen Ressourcen und die Erschöpfung der Arbeitergesellschaft führen zu einer Gewichtsverlagerung der ökonomischen Wertschöpfung in nicht-industrielle Sektoren (Kübler, 2009). Dies drückt sich als Wandel von der Industriegesellschaft hin zur Dienstleistungs- wie Informationsgesellschaft aus, wobei mehrere konstituierende Merkmale dieses Wandels existieren. Die Verlagerung von Industrieberufen zu Dienstleistungsberufen gehört dabei zu den prägnantesten Merkmalen. So werden Qualifikations- und Anforderungsprofile spezifisch erhöht: Die Nachfrage 
nach stark qualifizierten, (hoch) gebildeten Arbeitskräften steigt und der Fokus liegt nunmehr bei wissensintensiver Arbeit, die (noch) nicht von intelligenten, integrierten Systemen übernommen werden kann (Apt, Peter, von Stokar, Pärli \& Bovenschulte, 2014; Kübler, 2009; Schwahn et al., 2018).

Die Verschiebung hin zur wissensintensiven Arbeit fordert kommunikative Wege des Wissens- und Informationsaustauschs. Zeitschriften bieten grundsätzlich eine Möglichkeit des professionalisierten Wissensaustauschs zwischen Berufsangehörigen eines spezifischen Sektors und leisten somit einen relevanten Beitrag im Sinne von Weiterqualifikations- und Orientierungsfunktionen.

Im Schweizer Kontext sind sogenannte Tertiarisierungsprozesse (Abwanderung von Berufstätigen in Dienstleistungsberufe auch in Nicht-Dienstleistungssektoren) seit den 1970er Jahren beobachtbar, vor allem im landwirtschaftlichen Sektor ${ }^{2)}$. Seit den 1990er Jahren ist zudem ein Gewinn von Erwerbsanteilen bei Gesundheits-, Lehr- und Kulturberufen und den Berufen des Managements, der Administration, des Bank- und Versicherungsgewerbes und des Rechtswesens zu verzeichnen, während die Erwerbsanteile in den Produktionsberufen der Industrie und im Gewerbe (exkl. Bau) immer stärker abnehmen (Nathani, Hellmüller, Rieser, Hoff \& Nesarajah, 2018; Sheldon, 2005).

\section{These 5: Zeitschriftenthemen, Fokus Beruf}

Es wird angenommen, dass der Zeitschriftenmarkt auf diese berufsstrukturellen Entwicklungen reagiert. Die Zunahme von Berufsfeldern und Berufsbildern führt zu einer Ausweitung von Angeboten aus dem und für den Dienstleistungssektor im Zeitschriftenmarkt.

\section{Methode \& Design}

Ziel dieser Analyse ist es, Strukturmerkmale bezüglich der Themen, der Absender und des Zwecks von Zeitschriften in diachroner Perspektive in der Schweiz zu erfassen und damit die Thesen zu prüfen. Grundannahme ist, dass sich die Ausdifferenzierung und Pluralisierung sozialer Gruppen im Zeitschriftenangebot widerspiegelt.

Zeitschriften werden dabei definiert als regelmäßig über einen längeren Zeitraum erscheinende, meist gedruckte Publikationen, mit einem oder mehreren thematischen Schwerpunkten und spezifischen Darstellungsweisen (Hintergrundinformationen, Visualisierung), die prinzipiell jedem zugänglich sind, sich jedoch meist an ein spezifisches Zielpublikum richten (Rautenberg, 2015; Winter, 2004). Sie dienen - anders als Zeitungen, deren primäre Leistung auf das Selektieren, Einordnen und Spiegeln aktueller gesamtgesellschaftlicher Prozesse gerichtet ist - vor allem der Bereitstellung von wie dem Austausch über Themen von spezifischen Interessen.

Ein Großteil der Publikums-Zeitschriften (kommerzielle Zeitschriften) in der Schweiz werden von den umsatzstärksten Presseverlagen (Ringier AG, Tamedia AG, NZZ Gruppe, AZ Medien AG) publiziert. Diese Verlage vertreiben allerdings nicht ex-

2) Diese Aussagen basieren auf den Ergebnissen der Schweizerischen Arbeitskräfteerhebung (SAKE), der Schweizerischen Volkszählung (1850-2000) und der 5chweizerischen Strukturerhebung (1990-heute). (www.bfs. admin.ch). 
klusiv Zeitschriften, sondern auch einen Großteil der Schweizer Zeitungen (Verband Schweizer Medien, 2016). Fachzeitschriften (Berufszeitschriften, wissenschaftliche Zeitschriften) werden meist von kleineren (Fach-)Verlagen wie z.B. dem Dike Verlag, dem Schweizerischen Ärzteverlag EMH oder dem Walter Verlag publiziert. Diese produzieren ebenfalls nicht zwingend ausschliesslich Zeitschriften, sondern auch anderwärtige (Fach-) Literatur. Ebenso existieren in der Schweiz einige berufs-, fach- oder themenspezifische Vereine und Verbände, die ihre eigenen Zeitschriftentitel vertreiben und nicht als Verlag oder Medienunternehmen zu verzeichnen sind. Dies gilt auch für Unternehmen (z.B. Coop, Migros, Implenia etc.), die für ihre Mitarbeiter sowie auch für ihre Kunden Zeitschriften publizieren. Zeitschriften tragen über Inserierungs- und Werbeplätze zu einem erheblichen Teil zum Gesamtwerbemarkt bei. Der Netto-Werbeumsatz ist bei den Publikums-, Finanz- $\&$ Wirtschaftszeitschriften (ca. 300 Mio. im Jahr 2016) am höchsten und mehr oder weniger am stabilsten, was insofern Sinn macht, als dass kommerzielle Publikumszeitschriften die „passenden“ Werbeflächen bieten und eine höhere Reichweite als Spezial- oder Fachzeitschriften verzeichnen. Bei den Spezial- sowie Fachzeitschriften ist der Umsatz (bei beiden ca. 80 Mio. im Jahre 2016) seit ca. 2009 konstant gesunken (Stiftung Werbestatistik, 2017). Im Schweizer Zeitschriftenmarkt verzeichnet die Spezialpresse allerdings durchgängig die höchsten und stabilsten Auflagenzahlen (von 2008 bis 2018 zwischen 3.8 Mio. und 3 Mio.) in Relation zur Publikums-, Finanz-, und Wirtschaftspresse (von 2008 bis 2018 zwischen 2 Mio. und 1.3 Mio.) und der Fachpresse (von 2008 bis 2018 zwischen 0.5 Mio. und 1.8 Mio.). Bei der Fachpresse gab es einen signifikanten Anstieg von ca. 0.4 Mio. 2012 zu ca.1.7 Mio. Auflagen in 2014 (WEMF, 2019; beglaubigte Auflagezahlen).

Zur Beantwortung der zentralen Fragestellungen und Prüfung der Thesen wurde auf eine repräsentative Strukturanalyse der in den Titellisten des Katalogs der Schweizer Presse sowie ergänzend der Datenbank Helveticat der Schweizerischen Nationalbibliothek geführten Zeitschriftentitel zurückgegriffen. Der Katalog der Schweizer Presse wurde vom Verband Schweizerischer Werbegesellschaften herausgegeben und erfasste Basisdaten von Printpublikationen für die Platzierung von Inserate-Werbung. Der Katalog beinhaltet daher nur Zeitschriften, die auch oder vor allem ein kommerzielles Interesse durch den Verkauf von Werbung haben. Die darin gelisteten Zeitschriften können daher nicht als vollkommen repräsentativ für den gesamten Zeitschriftensektor gelten.

Analysiert wurde eine repräsentative Stichprobe der gelisteten Zeitschriftentitel pro $\mathrm{Jahr}^{3)}$ - soweit möglich - im Dreijahresrhythmus zwischen 1975 und 2016. Nachfolgender Tabelle kann die Grundgesamtheit $(\mathrm{N}=21$ '889) sowie der kalkulierte Stichprobenumfang $(n=4531)$ entnommen werden. Nach der Sichtung des Materials beläuft sich die tatsächlich realisierte Stichprobe auf $n=3977$, da einige Zeitschriftentitel bzw. einige Jahrgänge von Zeitschriften nicht mehr auffindbar waren.

3) Somit sind in der gesamten Stichprobe Mehrfach-Nennungen von Zeitschriftentiteln möglich. 
Tabelle 1: Grundgesamtheit und kalkulierte Stichprobe ${ }^{4)}$

\begin{tabular}{|c|c|c|c|c|}
\hline Jahr & \multicolumn{2}{|c|}{ Anzahl Zeitschriften } & \multicolumn{2}{|c|}{ Umfang kalkulierte Stichprobengröße } \\
\hline & Katalog Pub & Katalog Fach & Katalog Pub & Katalog Fach \\
\hline 1975 & 42 & 1044 & 37 & 216 \\
\hline 1977 & 47 & 1071 & 41 & 217 \\
\hline 1979 & 47 & 792 & 41 & 202 \\
\hline 1981 & 48 & 1360 & 41 & 226 \\
\hline 1984 & 57 & 1035 & 48 & 215 \\
\hline 1987 & 38 & 1632 & 34 & 233 \\
\hline 1989 & 37 & 1280 & 33 & 224 \\
\hline 1991 & 43 & 1275 & 38 & 224 \\
\hline 1994 & 36 & $1007^{*}$ & 32 & 214 \\
\hline 1996 & 35 & $1037^{*}$ & 32 & 215 \\
\hline 1998 & 33 & $1004^{*}$ & 30 & 214 \\
\hline \multicolumn{5}{|c|}{ Katalog Gesamt } \\
\hline 2000 & & & & \\
\hline 2002 & & & & \\
\hline 2004 & & & & \\
\hline 2006 & & & & \\
\hline 2008 & & & & \\
\hline 2010 & & & & \\
\hline \multicolumn{5}{|c|}{ Helveticat } \\
\hline 2012 & & & & \\
\hline 2014 & & & & \\
\hline 2016 & & & & \\
\hline Gesamt & & & & \\
\hline
\end{tabular}

Im Rahmen der Strukturanalyse wurden folgende Merkmale erhoben (Reliabilitätswerte - Krippendorff's Alpha - in Klammern).

- Absender (.94): Die Ausdifferenzierung von Zeitschriftenabsendern wurde anhand einer Variable ermittelt, welche die redaktionelle Verantwortung verschiedenen Akteuren zuordnet. Folgende Akteure werden innerhalb dieser Kategorie als Absender verstanden: Verlage/Medienunternehmen, Non-Profit-Unternehmen (Verbände/Vereine, NGO/NPO/Stiftungen, Parteien, Kirchen/kirchlich-soziale Vereinigungen, öffentliche/staatliche Organisationen/Einrichtungen), Unternehmen (kein Verlag).

- Ziel/Zweck (.90): Ziel dieser Variable ist es, zu bestimmen, zu welchem Zweck eine Zeitschrift publiziert wird. Zwischen folgenden Zielen wird hier unterschieden: Binnenkommunikation mit Mitgliedern/Mitarbeitern (interne Kommunikation), Binnenkommunikation zwischen Angehörigen eines Berufszweiges (nicht an Betriebe

4) Von 1975-1998 publizierte der Verband Schweizer Werbegesellschaften (VSW) den Katalog der Schweizer Presse in zweifacher Ausführung: (1) als Katalog der Publikums-und Spezialzeitschriften (Katalog Pub) und (2) als Katalog der Fachzeitschriften (Katalog Fach). Ab 2000 wurden diese beiden Versionen zusammengelegt (Katalog Gesamt). Die Publikation des Katalogs der Schweizer Presse wurde 2010 gänzlich eingestellt, so dass für die Jahre 2012-2016 auf die Zeitschriftendatenbank Helveticat der Schweizerischen Nationalbibliothek (SNB) zugegriffen werden musste. 
etc. gebunden), Außenkommunikation mit Kunden, nicht primär kommerzielles Interesse (Informieren steht im Vordergrund, Vermittlung von Weltanschauung/Lebensweisen, kein Profit), primär kommerzielles Interesse/Profit-orientiert.

- Themenbereich (.93): Die Ausdifferenzierung von Themenbereichen wurde anhand einer Themenliste (ca. 70 Themen), die induktiv und deduktiv erstellt wurde, gemessen. Vor allem das induktive Vorgehen führte dazu, dass sich die Ausprägungen der einzelnen Variablen auf teilweise unterschiedlichen Abstraktionsniveaus befinden: So wurden bei der Kategorienentwicklung an einem Teil des Analysematerials Zeitschriften mit sehr globalen Themenkategorien wie Politik \& Gesellschaft als auch sehr detaillierten Themenfoki wie «Fischen/Angeln» identifiziert. Anhand dieser Variable soll ermittelt werden, zu welchem Themenschwerpunkt eine Zeitschrift zugeordnet werden kann. Es waren nur einfache Zuschreibungen möglich, um klare Zuteilungen zu garantieren. Codiert wurden bei mehreren Schwerpunkten die erst genannten. Die Kategorie beinhaltet u.a. die Ausprägungen Politik \& Gesellschaft, Verwaltung, Sicherheit/Militär, Wirtschaft/Finanzen, Beruf (Primär-, Sekundär-, Tertiär-, Quartiär-, Quintorsektor), Arbeit/Gewerkschaften/Büro; Religion/Esoterik, Sport (Fussball, Tennis, Golf, Volleyball, etc.), Freizeit (Kunst, Kultur, Musik, Mode, Lifestyle, Gesundheit, Wellness, Literatur, Sammeln, Auto/Motor, Computer/Technik etc.), Wissenschaft, Natur/Umwelt/Tier;...

Vor allem die Variablen Zweck und Absender stehen dabei in einem engen Zusammenhang: Ist der Absender ein Medienunternehmen, so stehen auch meist kommerzielle Zwecke im Vordergrund. Wird die Zeitschrift von einem Berufsverband herausgegeben, werden vor allem binnenkommunikative Ziele mit der Publikation verfolgt. Da die Auswertung nicht multivariat erfolgt, sondern jeweils nur die einzelnen Ausprägungen der Variablen betrachtet werden, stellt das für die vorliegende Analyse kein Problem dar.

\section{Ergebnisse der Analyse des Schweizerischen Zeitschriftenmarktes}

Nachfolgend werden die Ergebnisse der Zeitschriftenanalyse entlang der formulieren Thesen zum Zeitschriftenabsender, -zweck und Themenspektrum dargestellt und unter Berücksichtigung des Forschungsstandes diskutiert.

\subsection{Zur Differenzierung der Themenbereiche im Zeitschriftenmarkt}

Den Schwerpunkt der Analyse bildet die Überprüfung der Thesen zur thematischen Pluralisierung des Zeitschriftenmarktes. Es wird dabei angenommen, dass sich gesellschaftliche Differenzierungsprozesse maßgeblich auch in einer Differenzierung des Themenspektrums im Zeitschriftensektor zeigt. So wird, um es an einem Beispiel aufzuzeigen, angenommen, dass sich ein verstärktes Gesundheitsbewusstsein in der Gesellschaft in einer Vielzahl an Zeitschriften, die sich dieser Thematik widmen, widerspiegelt. Zum einen kann angenommen werden, dass Medienverlage aus ökonomi- 
schen Interessen Zeitschriften zu wahrgenommenen Trendthemen anbieten, um den (angenommenen) Bedürfnissen der Leser und damit eigenen ökonomischen Interessen nachzukommen. Zum anderen wird unterstellt, dass aus der Differenzierung von gesellschaftlichen Werten, Professionen, Interessen und Aktivitäten auch ein zunehmendes Bedürfnis nach spezifischen Wissen und Austauschoptionen entsteht, dem durch die Gründung von Zeitschriften nachgekommen wird. Zur Identifikation spezifischer Themenmuster und -trends wird zunächst das aggregierte Themenspektrum gesamthaft dargestellt. Mit Kapitel 4.4 und 4.5 erfolgt eine „Nahaufnahme“ der Entwicklungen der Themen „Freizeit" und „Beruf“.

Nachfolgender Abbildung 1 lässt sich die Entwicklung der aggregierten Zeitschriftenthemen für den Erhebungszeitraum (1975-2016) erkennen. Unterschieden wurde zwischen Zeitschriften zum Themenbereich Beruf (Bsp. Psychoscope - Magazin für Psychologie), Wissenschaft \& Bildung (Bsp. Schweizerische Zeitschrift für Erziehungswissenschaft), Freizeit \& Sport (Bsp. Style), Tier \& Natur (Bsp. Welt der Tiere), Politik \& Gesellschaft (Bsp. Frau ohne Herz: die feministische Lesbenzeitschrift) sowie Familie \& Ehe (Bsp. Schweizer Illustrierte). Es wird deutlich, dass der Anteil an Zeitschriften zu Freizeitthemen mit Ausnahme des letzten Erhebungsjahrs 2016 kontinuierlich ansteigt. Zeitschriften, die berufliche Themen besprechen, verlieren hingegen prozentual im Zeitverlauf an Bedeutung. Dem gesteigerten Freizeitverhalten und -bedürfnis (Franzen \& Vogl, 2013; Hug \& Kriesi, 2010; Inglehart, 2018; Kübler, 2009; Lüdtke, 1996) scheint damit Rechnung getragen zu werden.

Abbildung 1: Anteil Zeitschriftentitel nach aggregierten Themenbereichen (\%)

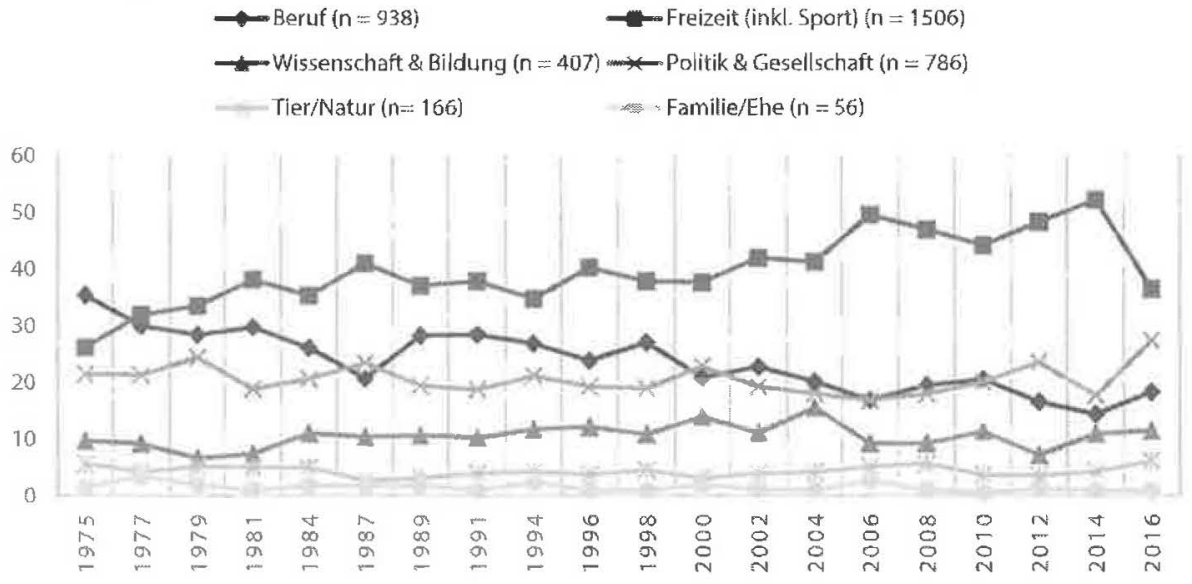

Anmerkung: 110 Zeitschriften nicht zuordenbar; $N=3859$

\subsection{Zur Differenzierung der Absender von Zeitschriften}

Das Erkenntnisinteresse der Studie gilt der Frage, ob sich Veränderungen bei den Zeitschriftenquellen respektive bei den Akteuren, die Zeitschriften herausgeben, im Zeit- 
verlauf darstellen lassen. Grundthese ist, dass diachron betrachtet ein Diversifizierungsprozess einsetzt, in dem eine Vielzahl unterschiedlicher Akteure zunehmend im Zeitschriftensektor aktiv sind. Neben privaten Medienunternehmen, wurden auch wachsende Aktivitäten von anderen Unternehmen und Organisationen des Non-ProfitBereichs erwartet.

Abbildung 2: Anteil Zeitschriften-Absender (aggregiert) (\%)

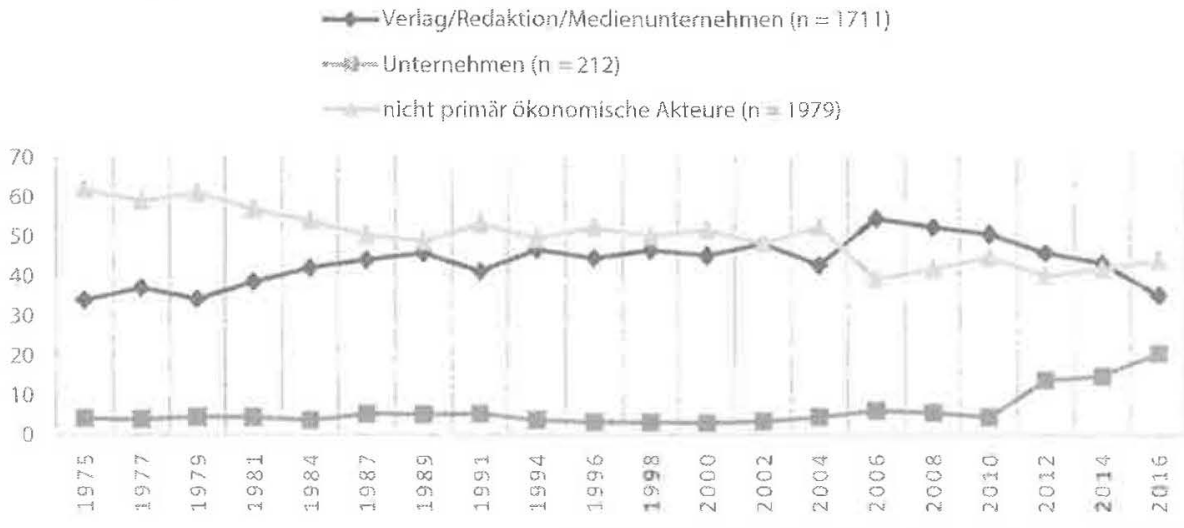

Anmerkung: 75 Zeitschriften nicht zuordenbar; $N=3902$

Wie Abbildung 2 zeigt, ist in Übereinstimmung mit These 2 im Zeitverlauf ein Differenzierungstrend bei den Absendern erkennbar: Unterschiedliche Akteurtypen zeichnen sich zunehmend für Zeitschriften verantwortlich. Ab den 2010er Jahren treten verstärkt Unternehmen, wie bspw. die Detailhandelskette Migros, als Herausgeber von Zeitschriften auf, die dieses Medium als Mittel der Kundenbindung und -werbung erkannt zu haben scheinen. Der klassische Medienverlag wie bspw. Ringier Axel Springer hat bis Mitte der 2000er Jahre im Trendverlauf als Absender von Zeitschriften an Bedeutung gewonnen. Seitdem ist jedoch ein deutlicher Rückgang von Verlagen als Herausgeber von Zeitschriften zu erkennen. Möglicherweise ist dies mit einem «Abwandern» kommerzieller Inhalte in den Onlinebereich zu erklären, der als Kanal und vergleichsweise kostengünstige Werbefläche vor allem nach der Pressekrise zunehmend an Bedeutung gewinnt (Kradolfer, Custer \& Künzler, 2010, S. 33; Kopp, 2006; Stadel, 2016; Studer, 2017). Zu Beginn der 70er Jahren engagierten sich mit einem Anteil von ca. 60 Prozent vor allem Absender, mit nur wenig oder keinen ökonomischen Motiven, als Herausgeber von Zeitschriften wie bspw. die Surprise NGO. Diese Bedeutung nimmt im Zeitverlauf jedoch deutlich ab. In den 2010er Jahren sind es nur noch rund 40 Prozent. Auch hier ist ein „Verlagern“ auf kostengünstigere Onlineplattformen anzunehmen.

\subsection{Zur Differenzierung des Zeitschriftenzwecks}

Die dritte These postuliert eine Heterogenisierung des Zwecks zur Herausgabe von Zeitschriften. Es wird angenommen, dass sich neben rein ökonomischen Zielstellun- 
gen über die Zeit auch die Binnenkommunikation zwischen Mitgliedern oder auch die Kundenbindung an Unternehmen und Dienstleistern als Hauptmotiv für die Publikation einer Zeitschrift feststellen lässt.

Ein eindeutiger linearer Trend zur Diversifizierung des Zeitschriftenzwecks ist jedoch nicht festzustellen (vgl. Abb. 3): Bereits zu Beginn des Erhebungszeitraums in den 70er Jaren waren sowohl kommerzielle als auch Vernetzungsmotive (Binnenkommunikation zwischen Mitgliedern einer Organisation wie bspw. das Touring-Magazin vom TCS Schweiz und zwischen Angehörigen einer Profession wie die Schweizerische Ärztezeitung) ausschlaggebend für die Zeitschriftenherausgabe.

Erkennbar sind dabei analoge Entwicklungen zur Heterogenisierung der Absender (siehe Ergebnisse zu These 2):

- Vergleichbar mit dem Rückgang der Medienverlage als Zeitschriftenquelle ist hier auch eine Verringerung rein kommerzieller Zwecke zur Publikation von Zeitschriften ab Mitte der 2000er Jahre zu erkennen.

- Auch dass Unternehmen als Absender zunehmend an Bedeutung gewinnen, zeigt sich in einer zunehmenden Nutzung der Zeitschrift zur Außenkommunikation mit Kunden seit den 2010er Jahren (Weichler, 2014).

- Die Bedeutung der Zeitschrift zu beruflichen Binnenkommunikation hat abgenommen. Möglicherweise wird hier auch verstärkt auf Onlinetools (Chat, Foren, Blogs) zurückgegriffen.

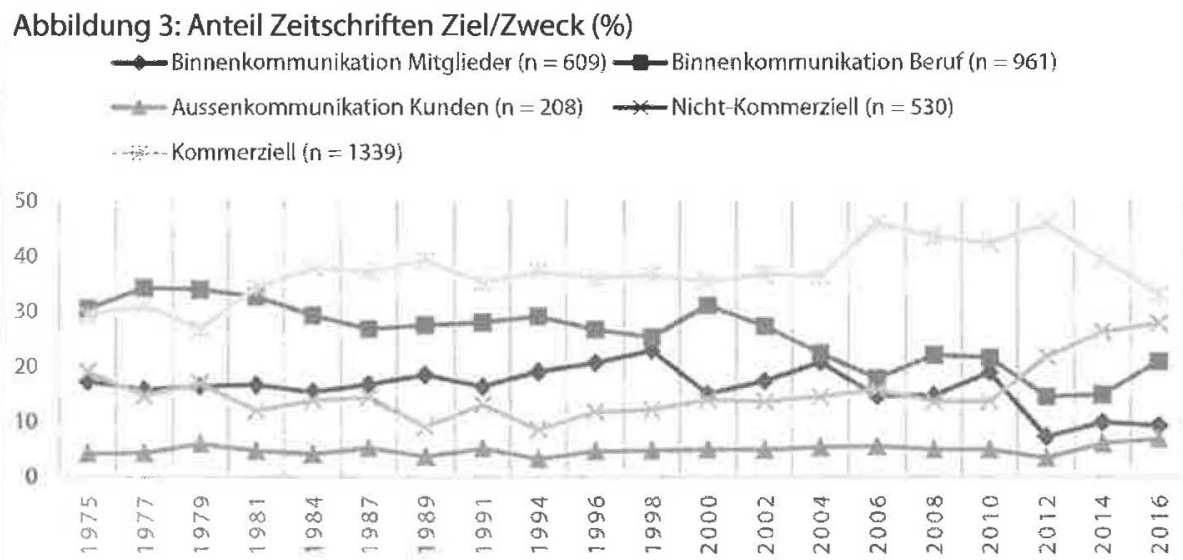

Anmerkung: 330 Zeitschriften nicht zuordenbar; $N=3647$

Kritisch anzumerken ist hierbei jedoch, dass nur Zeitschriften analysiert werden konnten, die im Katalog der Schweizer Presse gelistet sind, der bei nicht-kommerziellen Zeitschriften möglicherweise blinde Flecken aufweist.

\subsection{Zur Differenzierung der Zeitschriften im Freizeitbereich}

Wie die Analyse des aggregierten Themenspektrums aller Zeitschriften bereits erkennbar macht, spiegeln vor allem Zeitschriften im Freizeitbereich das gestiegene Bedürfnis 

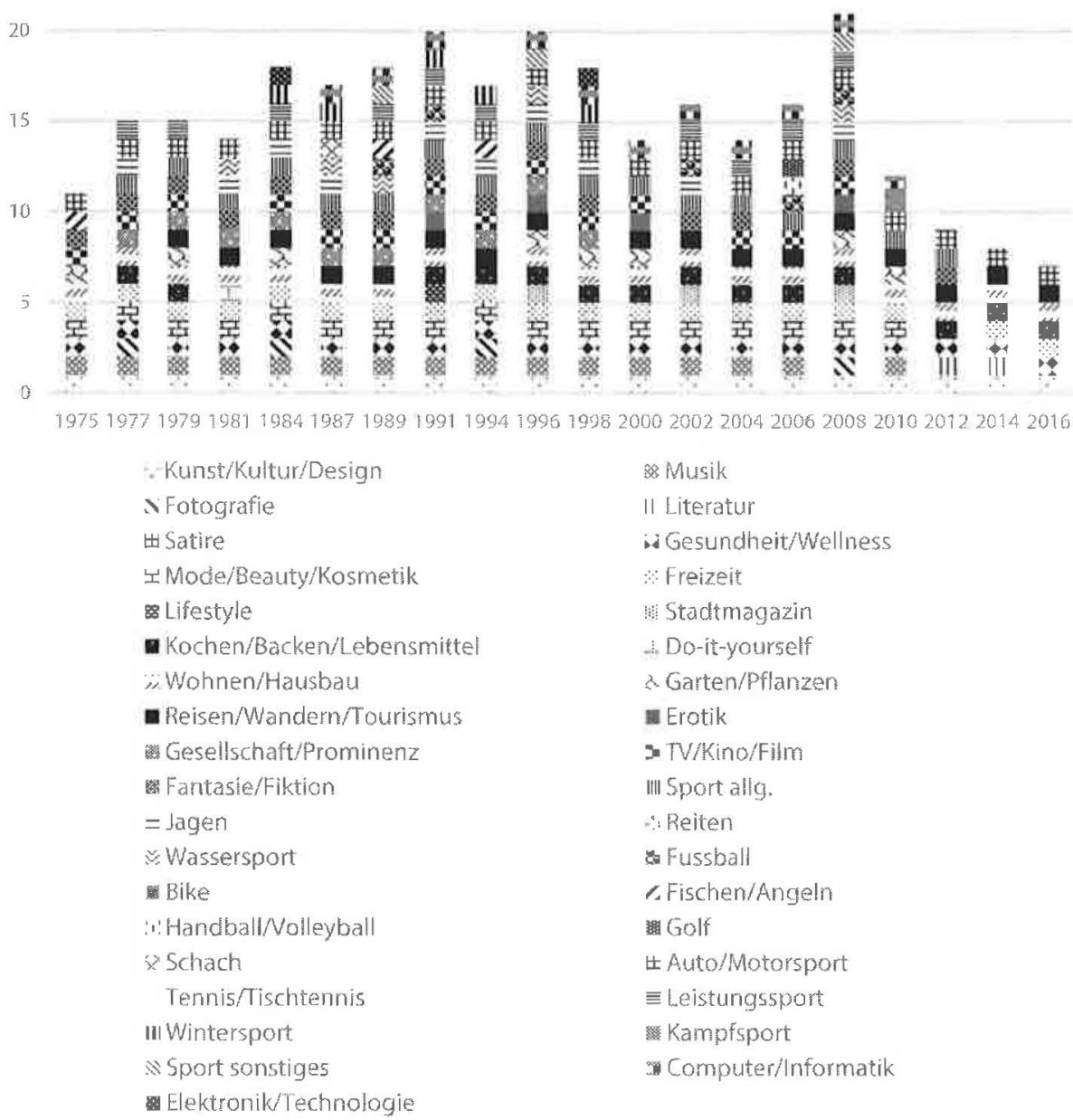

Anmerkung: Maximaler Differenzierungsgrad: 37 (37 Themen); $N=1506$

nach Verwirklichung und Befriedigung in diesem Bereich wider. Nachfolgend soll daher ein vertiefender Blick auf diesen Themenbereich gelegt werden, um prüfen zu können, inwiefern eine Heterogenisierung der Freizeitthemen beobachtet werden kann. Hierfür wurde ein Differenzierungsindex mit einem möglichen Maximalwert von 37 gebildet. Dieser Wert basiert auf den insgesamt 37 Freizeitthemen-Kategorien (z.B. Golf, Musik, Kochen, Reisen, Reiten etc.), zu welchen die Zeitschriften zugeordnet werden konnten. Um die Ausdifferenzierung auf der Ebene der Freizeitthemen zu ermitteln, wurde wie folgt vorgegangen: Pro Jahr wurde ermittelt, ob diese Themen vertreten sind oder nicht. Als vertreten galt ein Thema, wenn zwei oder mehr Zeitschriften dieser The- 
menkategorie vorkommen. Ein vertretenes Thema wurde mit dem Wert „1“ versehen. Hohe Balken bzw. Balken mit einem Wert nahe bei 37 sollen als Indiz für eine hohe Ausdifferenzierung von Themenbereichen gedeutet werden. Mit den schraffierten Felden soll angezeigt werden, welche Themenbereiche in welchen Jahren vorkommen.

Bis Mitte der 2000er Jahre ist ein Trend zur Themendifferenzierung innerhalb des Freizeitbereichs erkennbar: Während 1975 nur 11 Themenspektren im Freizeitbereich von mindestens zwei Zeitschriften abgedeckt wurden, waren es im Jahr 2008 bereits mehr als 20. Ab 2008 wird jedoch eine sehr deutliche Abnahme der Themenheterogenität erkennbar (vgl. Abb. 4). Auch dieser Befund lässt sich mit der neuen Onlinemedienumgebung plausibilieren: Statt möglicherweise ökonomisch nicht tragfähige Zeitschriften zu sehr spezifischen Freizeitthemen auf dem Printmarkt zu lancieren, nutzen Organisationen zunehmend Onlinekanäle, um über diese über Interessen und Aktivitäten zu informieren. Im Printbereich können sich dann solche Themenbereiche „halten“, die für einen vergleichsweise großen Rezipientenkreis von Interesse sind wie bspw. Gesundheit/Wellness, Kochen/Backen, Reisen/Wandern/Tourismus oder Auto/Motorsport. In diesem Bereich befinden sich dann zumeist als Marke etablierte Titel wie Du, Natürlich, Al Dente, Wandermagazin Schweiz können sich halten (Konsolidierung, starke Marken) (Bleis, 1996).

\subsection{Zur Differenzierung der Zeitschriften im beruflichen Bereich}

Literatur zum Berufs- und Professionenwandel verweist häufig auf die Zunahme des Dienstleistungssektors als neues Betätigungsfeld. Es wird daher mit These 4 angenommen, dass der Zeitschriftenmarkt auf diese berufsstrukturellen Entwicklungen reagiert und vor allem Zeitschriften veröffentlicht, die Angehörigen dieser Berufszweige von Nutzen sind. Zeitschriften zu Produktionsberufen (bspw. in der Landwirtschaft) sollten parallel dazu abnehmen. Abbildung 5 zeigt jedoch deutlich, dass hier kein Trend im Zeitschriftenbereich erkennbar ist.

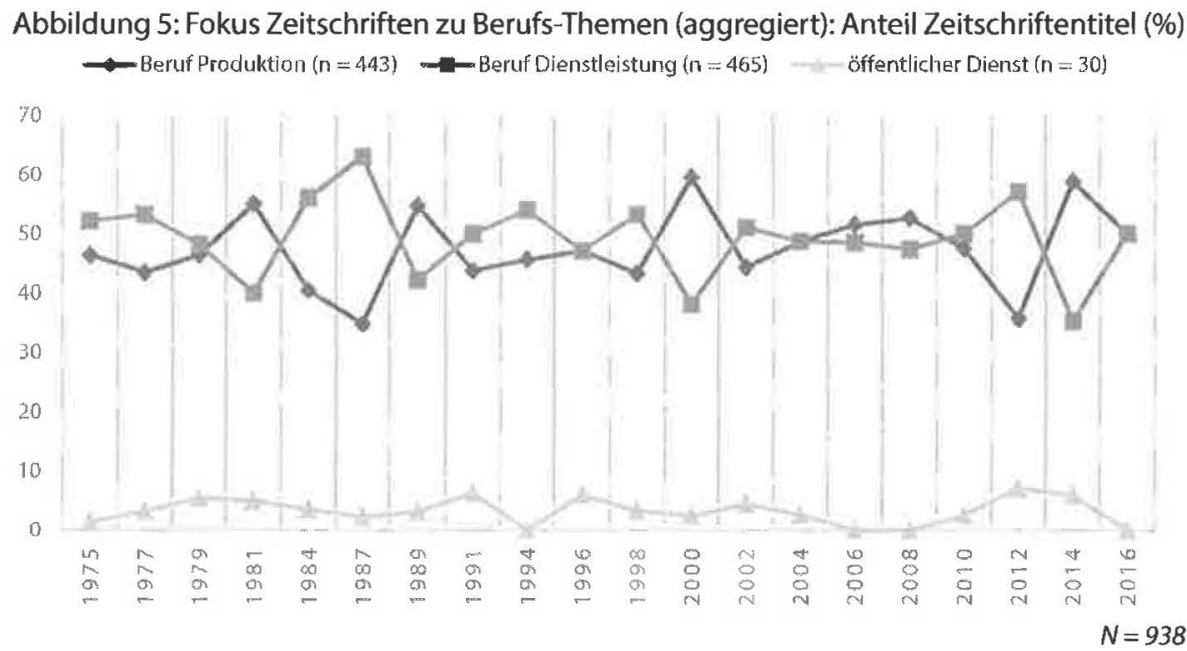




\section{Diskussion der Ergebnisse}

Gesellschaftliche Differenzierungsprozesse, so die zentrale Annahme des Beitrages, spiegeln sich in einem ausdifferenzierten Zeitschriftensektor wider, der den (neu entstandenen) gesellschaftlichen Bedürfnissen aus ökonomischen oder sozialen Erwägungen Rechnung trägt. Ziel des Beitrages war es, diese Annahme anhand einer diachronen Strukturanalyse des Schweizer Zeitschriftensektors mit Blick auf die Entwicklung der Absender, Zwecke und Themen der Zeitschriften zu prüfen.

Die Analyse zeigte dabei keine eindeutige Differenzierungsrichtung: Während eine Diversifizierung der Absender im Zeitverlauf bejaht werden kann, müssen die formulierten Differenzierungsthesen für die Zwecke und Themen der Zeitschriften mangels eindeutiger Trendlinien teilweise abgelehnt werden. Lediglich eine Ausdifferenzierung der Themen im Freizeitbereich ist nachweisbar. Für die fehlende eindeutige Differenzierung im Zeitschriftensektor können folgende Gründe als ursächlich angesehen werden:

- Es besteht die Möglichkeit, dass der gewählte Analysezeitraum zu kurz für tatsächlich ablesbare Differenzierungstrends ist. Im Sinne der Interessenskommunikation (Verbände, Parteien etc. als Absender) könnte das besonders zutreffen, da diese Form der Kommunikation über Zeitschriften schon lange vor den 1970er existiert hat (Stöber, 2002).

- Methodisch besteht die Möglichkeit, dass in Bezug auf die Ausdifferenzierung der Themen die Themenkategorien zu wenig stark differenziert abgefragt wurden, so dass auf einer zu hoch aggregierten Ebene Differenzierung untersucht wurde.

- Dass keine klaren Differenzierungstrends aufgezeigt wurden, kann auch an einer trägen Reaktionszeit des Zeitschriftenmarktes auf gesellschaftliche Differenzierungsprozesse liegen. So könnten Verlage bestrebt sein, innerhalb von Titeln neue Angebote zu machen, aber nicht sogleich neue Titel auf den Markt zu bringen. Dazu gehört auch, dass Verlage mit Einzeltiteln den Markt sondieren können.

- Es ist also denkbar, dass die angenommene Widerspiegelung von Differenzierungsdynamiken bei Zeitschriften nicht auf der Ebene der Zeitschriftentitel erfolgt, sondern auf der Ebene der Zeitschrifteninhalte. Dahinter liegt die Vermutung, das thematische Unterschiede auf der Titelebene durch die Konsolidierung (Bündelung bzw. Zusammenlegung von Zeitschriftentiteln, sowie Beilagen-Hefte etc.) bzw. Etablierung von Titeln im Zeitschriftenmarkt nicht zwingend erkennbar sind. Diese Titel unterscheiden sich bzw. differenzieren sich aber inhaltlich.

Die bisherig genannten Gründe, warum keine Differenzierungstrends aufgezeigt wurden, basieren auf der Vorannahme, dass ein linearer Differenzierungstrend erkennbar sein müsste. Diese Vorannahme kann aber grundsätzlich hinterfragt werden, da Differenzierungsdynamiken nicht zwingend linear, sondern in einem Wandel von Ausdifferenzierung und Konsolidierung ablaufen müssen. In diesem Sinne weisen die Ergebnisse vor allem auf die starke Mikrovariabilität des Marktes hin. 
In der Zeitschriftenforschung allgemein besteht das Grundproblem, dass der Grundbestand von Zeitschriften grundsätzlich unbekannt ist und bleibt. Dieses Problem könnte auch ein Grund für die fehlenden Differenzierungstrends in den vorgestellten Ergebnissen sein. Wie bereits erwähnt, werden zwar Zeitschriftentitel-Listen wie die des Katalogs der Schweizer Presse geführt, diese beinhalten allerdings meistens nur jene Zeitschriften, die für den Werbe- bzw. Inserierungsmarkt relevant sind. Es gibt also eine „Dunkelziffer" von vor allem nicht kommerziellen Zeitschriften, die keine besondere Marktrelevanz haben, aber dennoch publiziert werden. Dies wirft einerseits die Frage auf, wie geeignet solche Listen für ähnliche Forschung sind und anderseits wie dieses Quellen-Problem gelöst werden kann.

Unsere Befunde legen einen Einfluss der neuen Onlinemedienumgebung nahe. So scheint es plausibel anzunehmen, dass den Bedürfnissen der differenzierten Gesellschaft zunehmend mit vergleichsweise kostengünstigen, einfach zugänglichen und jederzeit abrufbaren Möglichkeiten des Internets nachgegangen wird. Das Medium gedruckte Zeitschrift scheint daher als Spiegel von gesellschaftlichen Differenzierungsprozessen an Bedeutung verloren zu haben. Gemäß Riepl'schem Gesetz (Meier, 2014) dürfte jedoch zu erwarten sein, dass die Zeitschrift - auch als gedrucktes Medium nicht einfach verschwindet, sondern sich anpasst und weiterentwickelt. Dies zu analysieren und zu erklären, ist Aufgabe zukünftiger Forschungsvorhaben.

\section{Literatur}

Apt, W., Peter, M., von Stokar, T., Pärli, K. \& Bovenschulte, M. (2014). Der Wandel der Arbeitswelt in der Schweiz. Gesellschaftliche, strukturelle und technologische Entwicklungen. it perspektive, 20, 1-12.

Aubrey, J.S. \& Hahn, R. (2016). Health Versus Appearance Versus Body Competence: A Content Analysis Investigating Frames of Health Advice in Women's Health Magazines. Journal of Health Communication, 21(5), 496-503.

Babic, D. \& Jandura, O. (2017). Ein Blick in die Vergangenheit. Die Fragmentierung des Publikums von Zeitschriften. In O. Jandura, M. Wendelin, M. Adolf, \& J. Wimmer (Hg.), Zwischen Integration und Diversifikation. Medien und gesellschaftlicher Zusammenhalt im digitalen Zeitalter (S. 105-119). Wiesbaden, DE: Springer.

Bazzini, D.G., Pepper, A., Swofford, R. \& Cochran, K. (2015). How Healthy are Health Magazines? A Comparative Content Analysis of Cover Captions and Images of Women's and Men's Health Magazines. Sex Roles, 72(5-6), 198-210.

Beetham, M. (1996), A Magazine of Her Own? Domesticity and Desire in the Woman's Magazine, 1800-1914. London, UK: Routledge.

Berger, P.A. (1986). Entstrukturierte Klassengesellschaft? Wiesbaden, DE: Verlag für Sozialwissenschaften.

Bleis, T. (1996). Erfolgsfaktoren neuer Zeitschriften. Empirische betriebswirtschaftliche Untersuchung zur Entwicklung und Markteinführung von Publikumstiteln. München, DE: Fischer.

Blöbaum, B. (1994). Journalismus als soziales System. Geschichte, Ausdifferenzierung und Verselbstständigung. Opladen, DE: Westdeutscher Verlag.

Blöbaum, B. (2000). Strukturwandel des Journalismus - Strukturwandel von Öffentlichkeit. In O. Jarren, K. Imhof, \& R. Blum (Hg.), Zerfall der Öffentlichkeit? (S.127-138). Konstanz, DE: UVK.

Bohrmann, H. \& Schneider, P. (1975). Zeitschriftenforschung. Ein wissenschaftsgeschichtlicher Versuch. Berlin, DE: Verlag Volker Spiess. 
Bohrmann, H. (2002). Über Zeitschriftenforschung als T'eil der Publizistik- und Kommunikationswissenschaft. In A. Vogel \& C. Holtz-Bacha (Hg.), Publizistik: Sonderheft 3/2002, Zeitschriften und Zeitschriftenforschung (S. 28-41). Wiesbaden, DE: Westdeutscher Verlag.

Bürklin, W.P. (1988). Wählerverhalten und Wertewandel. Wiesbaden, DE: VS Verlag für Sozialwissenschaften.

Burzan, N., Lökenhoff, B., Schimank, U. \& Schöneck, N.M. (2008). Das Publikum der Gesellschaft. Inklusionsverhältnisse und Inklusionsprofile in Deutschland. Wiesbaden, DE: VS Verlag für Sozialwissenschaften.

Cauers, C. (2009). Mitarbeiterzeitschriften heute. Flaschenpost oder strategisches Medium? Wiesbaden, DE: VS Verlag für Sozialwissenschaften.

Davalos, D.B., Davalos, R.A. \& Layton, H.S. (2016). Content Analysis of Magazine Feadlines. Feminism er Psychology, 17(2), 250-258. doi: 10.1177/0959353507076559.

Dernbach, B. (2010). Die Vielfalt des Fachjournalismus, Eine systemalische Einführung. Wiesbaden, DE: VS Verlag für Sozialwissenschaften.

Donges, P. \& Jarren, O. (2017). Politische Kommunikation in der Mediengesellschaft. Wiesbaden, DE: Springer Fachmedien.

Fiedler, S. (2003). Sprachspiele im Comic. Das Profil der deutschen Comic-Zeitschrift Mosaik. Jeipzig, DE: Leipziger Universiiäts-Verlag.

Franzen, A. \& Vogl, D. (2013). Zeitpräferenzen und Umweltbewusstsein. Analysen mit dem Schweizer ISSP 2010. Schweizerische Zeitschrift für Soziologie, 39(3), 441-464.

Gerlach, P. (1988). Buchwissenschaftliche Beiträge aus dem Deutschen Bucharchiv München: Band 33. Zeitschriftenforschung. Probleme und Lösungsansälze dargestellt am Beispiel Journalism Quarterly (1964-1983). Wiesbaden, DE: Otto Harrassowitz.

Hasebrink, U. \& Domeyer, H. (2010). Zum Wandel von Informationsrepertoires in konvergierenden Medienumgebungen. In M. Harımann \& A. Hepp (Hg.), Die Mediatisierung der Alltagswelt (S. 49-64). Wiesbaden, DE: VS Verlag für Sozialwissenschaften.

Heinrich, J. (2002). Ökonomische Analyse des Zeitschriftensektors. In A. Vogel \& C. Holtz-Bacha (Hg.), Publizistik: Sonderheft 3/2002. Zeitschriften und Zeitschriftenforschung (S. 60-82). Wiesbaden, DE: Westdeutscher Verlag.

Hermes, J. (2010). Reading Women's Magazines. An Analysis of Everyday Media Use. Cambridgz, UK: Polity Press.

Hillmann, K. (2001). Zur Wertewandelforschung: Einführung, Übersicht und Ausblick. In G.W. Oesterdiekhoff \& N. Jegelka (Hg.), Werte und Wertewandel in westlichen Gesellschaften (S. 15-39). Wiesbaden, DE: VS Verlag für Sozialwissenschaften.

Hinnant, A. (2009). Getting the Science Right: An Experiment in How Readers Evaluate Medical News Coverage in Magazine Health Journalism. Journal of Health and Mass Communication, 1(1/2), 58-76.

Hoffmann, S. (2002). Archiv für Begriffsgeschichte: Sonderheft. Geschichte des Medienbegriffs. Hamburg, DE: Felix Meier Verlag.

Holmes, T (2007). Mapping The Magazine. Journalism Studies, 8(4), 510-521. doi: 10.1080/1461670070 1411714.

Hradil, S. (2002). Vom Wandel des Wertewandels. Die Individualisierung und eine ihrer Gegenbewegungen. In W. Glatzer, R. Habich, \& K.U. Mayer (Hg.), Sozialer Wandel und gesellschaftliche Dauerbeobachtung (S. 31-48). Wiesbaden, DE: VS Verlag für Sozialwissenschaften.

Hug, S, \& Kriesi, H. (2010). Value Change in Switzerland. Lanham, MD, USA: Lexington Books.

Informationsgemeinschaft zur Feststellung der Verbreitung von Werbeträgern e.V. (IVW). (2018). Auflagezahlen des 4. Quartals 2017. Abgerufen unter https:/www.ivw.eu/print/quartalsauflagen/pressemitteilungen/auflagenzahlen-des-4-quartals-2017 (18.03.2019).

Inglehart, R.F. (2018). Cultural Evolution. Cambridge, UK: Cambridge University Press.

Jentges, E., Brändli, M., Donges, P. \& Jarren, O. (2013). Communication of Political Interest Groups in Switzerland. Studies in Communication Sciences, 13(1), 33-40. 
Kern, T. (2011). Differenzierung als kreativer Prozess: die Herausbildung von Rollen in Publikumsnetzwerken. In T. Schwinn, C. Kronenberg, \& J. Greve (Hg.), Soziale Differenzierung (S. 285-304). Wiesbaden, DE: VS Verlag für Sozialwissenschaften.

Kohring, M. (2005). Wissenschaftsjournalismus. Forschungsüberblick und Theorieentwurf. Konstanz, DE: UVK.

Kopp, B. (2006). Hochschulschriften zur Betriebswirtschaftslehre: Band 144. Strategisches Marketing der Zeitungsverlage in Deutschland, Österreich und der Schweiz: Wege und Methoden zu strategischen Neuorientierung. München, DE: Utz.

Kösters, R. \& Jandura, O. (2018). Politische Kommunikation in heterogenen Lebenswelten. Kommunikationspraxis in politischen Milieus und Bedingungen ihrer Integration. SCM Studies in Communiation and Media, 7(2), 129-185.

Kradolfer, E., Custer, U. \& Künzler, M. (2010). Die wirtschaftliche Entwicklung der Medien in der Schweiz 2000-2010. Abgerufen unter https://www.bakom.admin.ch/bakom/de/home/suche.html\#die\%20wirtschaftlichen\%20entwicklungen (18.03.2019).

Kübler, H. (2009). Mythos Wissensgesellschaft. Gesellschaftlicher Wandel zwischen Information, Medien und Wissen: Eine Einführung. Wiesbaden, DE: VS Verlag für Sozialwissenschaften.

Kuhn, A. (2018). Zeitschriften und Medienunterhaltung. Zur Evolution von Medien und Gesellschaft in systemfunktionaler Perspektive. Wiesbaden, DE: Springer Fachmedien.

Lüdtke, H. (2000). Konsum und Lebensstile. In D. Rosenkranz \& N.F. Schneider (Hg.), Konsum. Soziologische, ökonomische und psychologische Perspektiven (S. 117-132). Wiesbaden, DE: VS Verlag für Sozialwissenschaften.

Luhmann, N. (1997). Die Gesellschaft der Gesellschaft. Frankfurt am Main, DE: Suhrkamp.

Luhmann, N. (2009). Die Realität der Massenmedien. Wiesbaden, DE: Springer Fachmedien.

Mathews, A.E., Laditka, S.B. Laditka, J.N. \& Friedman, D. (2009). What are the top-circulating Magazines in the United States Telling Older Adults about Cognitive Health? American Journa of Alzheimer's Disease and other Dementias, 24(4), 302-312.

Meier, U. (2014). 100 Jahre Riepl'sches Gesetz. In C. Kappes, J. Krone, \& L. Novy (Hg.), Medienwandel kompakt 2011-2013 (S.11-17). Wiesbaden, DE: Springer VS.

Nassehi, A. (2001). Moderne Gesellschaft. In G. Kneer, A. Nassehi, \& M. Schroer (Hg.), Klassische Gesellschaftsbegriffe der Soziologie (S. 208-245). München, DE: Fink.

Nassehi, A. (2004). Die Theorie funktionaler Differenzierung im Horizont ihrer Kritik. Zeitschrift für Soziologie, 33(2), 98-118.

Nathani, C., Hellmüller, P., Rieser, C., Hoff, O. \& Nesarajah, S. (2017). Ursachen und Auswirkungen des Strukturwandels im Schweizer Arbeitsmarkt. Rüschlikon, CH: Staatssekretariat für Wirtschaft SECO.

Neuberger, C. (2016). Journalismus und Medialisierung der Gesellschaft. In K. Meier \& C. Neuberger (Hg.), Journalismusforschung. Stand und Perspektiven (2., akt. u. erw. Auf.) (S. 341-371). Baden-Baden, DE: Nomos.

Nowak, D. (2009). Gesellschaft und Medien im Wandel. In S. Dierks (Hg.), Quo vadis Zeitschriften? Änderung der Medienlandschaft und Auswirkungen auf den Pressekäufer (S. 99-111). Wiesbaden, DE: VS Verlag für Sozialwissenschaften.

Peters, B. (1993). Die Integration moderner Gesellschaften. Frankfurt, DE: Suhrkamp.

Rautenberg, U. (2015). Reclams Sachlexikon des Buches. Von der Handschrift zum E-Book. Stuttgart, DE: Reclam.

Reckwitz, A. (2018). Die Gesellschaft der Singularitäten. Zum Strukturwandel der Moderne. Frankfurt am Main, DE: Suhrkamp.

Ringelmann, V. (1991). Gesundheit durch Zeitschriften. Hamburg, DE: Kovac,

Rössler, P. \& Ott, J. (2002). Tausend Mal das „erste Mal“. Kultiviert „Bravo“ jugendliche Vorstellungen von Sexualität? Eine empirische Studie. In A. Vogel \& C. Holtz-Bacha (Hg.), Publizistik: Sonderheft 3/2002. Zeitschriften und Zeitschriftenforschung (S. 169-195). Wiesbaden, DE: Westdeutscher Verlag. 
Röttger, U. (2002). Kundenzeitschriften: Camouflage, Kuckucksei oder kompetente Information? In A. Vogel \& C. Holtz-Bacha (Hg.), Publizistik: Sonderheft 3/2002. Zeitschriften und Zeitschriftenforschung (S. 109125). Wiesbaden, DE: Westdeutscher Verlag.

Sakamoto, K. (1999). Reading Japanese Women's Magazines: The Construction of New Identities in the 1970s and 1980s. Media Culture \&o Society, 21(2), 173-193.

Schimank, U. (1996). Theorien gesellschaftlicher Differenzierung. Opladen, DE: Leske + Budrich.

Schimank, U. (2001). Organisationsgesellschaft. In G. Kneer, A. Nassehi \& M. Schroer (Hg.); Klassische Gesellschaftsbegriffe der Soziologie (S. 278-307). München, DE: Fink.

Schimank, U. (2005). Die Entscheidungsgesellschaft. Komplexität und Rationalität der Moderne. Wiesbaden, DE: Verlag für Sozialwissenschaften.

Schlenker, J.A., Caron, S.L. \& Haltemann, W.A. (1998). A Teminist Analysis of Seventeen Magazine: Content Analysis from 1945 to 1995. Sex Roles, 38(1-2), 135-149.

Schmidt, J. H. (2008). Was ist neu am Social Web? Soziologische und kommunikationswissenschaftliche Grundlagen. In A. Zerfass, M. Welker \& J. Schmidt (Hg.), Neue Schriften zur Online Forschung: Band 1 Grundlagen und Methoden. Kommunikation, Partizipation und Wirkungen im Social Web: Von der Gesellschaft zum Individuum (S. 18-40). Köln, DE: Halem.

Schmolke, M. (2002). Kirchenpresse. In A. Vogel \& C. Holtz-Bacha (Hg.), Publizistik: Sonderheft 3/2002. Zeitschriften und Zeitschriftenforschung (S. 126-148). Wiesbaden, DE: Westdeutscher Verlag.

Scholl, A. \& Weischenberg, S. (1998). Journalismus in der Gesellschaft. Theorie, Methodologie und Empirie. Opladen, DE: Westdeutscher Verlag.

Schulze, G. (2005). Die Erlebnisgesellschaft. Kultursoziologie der Gegenwart (2.Aufl.). Frankfurt, DE; New York, NY: Campus Verlag.

Schwahn, F., Mai, C., \& Braig, M. (2018). Arbeitsmarkt im Wandel - Wirtschaftsstrukturen, Erwerbsformen und Digitalisierung. WISTA, 3, 24-49.

Seufert, W. (2004). Ökonomische Restriktion für die Ausdifferenzierung des Medienangebots am Beispiel des deutschen Zeitschriftenmarkts. In G. Siegert (Hg.), Schriften zur Medienwirtschaft und zum Medienmanagement: Band 6. Zwischenmarktversagen und Medienvielfalt. Medienmärkte im Fokus neuer medienökonomischer Anwendungen (S. 85-99). Baden-Baden, DE: Nomos.

Sheldon, G. (2005). Der Berufsstrukturelle Wandel der Beschäftigung in der Schweiz 1970-2000. Ausmass, Ursachen und Folgen. In Bundesamt für Statistik (BFS) (Hg.), Statistik der Schweiz, 3 Arbeit, Erwerb (S. 1-68). Neuchatel, CH: Bundesamt für Statistik.

Spellerberg, A. (1996). Soziale Differenzierung durch Lebensstile. Eine empirische Untersuchung zur Lebensqualität in West- und Ostdeutschland. Berlin, DE: Ed. Sigma.

Stadel, F. (2016). Verlage setzen bei Digitalstrategien auf eingeführte Namen und Labels. In S. Regier, H. Schunk \& T. Könecke (Hg.), Marken und Medien (S. 267-280). Wiesbaden, DE: Springer Fachmedien.

Stehr, A. \& Adolf, M. (2015). Ist Wissen Macht? Erkenntnisse über Wissen. Weilerswist, DE: Velbrück Wissenschaf.

Stephenson, S. (2007). The Changing Face of Women's Magazines in Russia. Journalism Studies, 8(4), 613-620.

Stiftung Werbestatistik Schweiz (2017). Werbeaufwand Schweiz 2017. Abgerufen unter https:/www.marketing.ch/wp-content/uploads/2018/02/Statistik_13.pdf (18.03.2019).

Stöber, R. (2002). Historische Zeitschriftenforschung heute. In A. Vogel \& C. Holtz-Bacha (Hg.), Publizislik: Sonderheft 3/2002. Zeitschriften und Zeitschriftenforschung (S. 42-59). Wiesbaden, DE: Westdeutscher Verlag.

Strassner, L. (1997). Zeitschrift. Tübingen, DE: Max Niemeyer Verlag

Studer, S. (2017). Pressestrukturen im Wandel. Eine organisationsökologische Analyse der Entwicklung schweizerischen Presseorganisationslypen und ihrer Angebote zwischen 1968 und 2013. In W.A. Meier (Hg.), Abbruch - Umbruch - Aufbruch: Globaler Medienwandel und lokale Medienkrisen (S. 45-68). Baden-Baden, DE: Nomos. 
Tschörtner, A. \& Schenk, M. (2009). Profil des deutschen Publikumszeitschriftenmarktes - eine Analyse der Entwicklungsbedingungen. In S. Dierks (Hg.), Quo vadis Zeitschriften? Änderung der Medienlandschaft und Auswirkungen auf den Pressekäufer (S. 19-44). Wiesbaden, DE: VS Verlag für Sozialwissenschaften.

Verband Deutscher Zeitschriftenverleger (VDZ) (2018). VDZ-Jahrespressekonferenz 2018. Abgerufen unter https://www.vdz.de/branche/branchendaten/ (18.03.2019).

Verband Schweizer Medien (VSM) (2016). Printmedienangebot in der Schweiz 2016. Abgerufen unter www,schweizermedien, ch (18.03.2019).

Vogel, A. (2002). Pressegattungen im Zeitschriftengewand. Warum die Wissenschaft eine Pressesystematik braucht. In A. Vogel \& C. Holtz-Bacha (Hg.), Publizistik: Sonderheft 3/2002. Zeitschriften und Zeitschriftenforschung (S. 11-27). Wiesbaden, DE: Westdeutscher Verlag.

Vogel, A. \& Holtz-Bacha, C. (2002). Vorwort. In A. Vogel \& C. Holtz-Bacha (Hg.), Publizistik: Sonderheft 3/2002. Zeitschriften und Zeitschriftenforschung (S. 7-8). Wiesbaden, DE: Westdeutscher Verlag.

Vogel, A. (2010). Zeitschriftenmarkt: WAZ-Gruppe schließt zu dominierenden Konzernen auf. Media Perspektiven, 6, 296-315.

Vogel, A. (2014). Publikumszeitschriften: lebhafte Marktentwicklung ohne Tendenzwende. Media Perspektiven, $6,347-371$.

Voll, P. (2001). Integration durch Differenz - Religion, Werte und Lebensstile in der Schweiz. In G. Pickel \& M. Krüggeler (Hg.), Religion und Moral (S. 135-168). Wiesbaden, DE: VS Verlag für Sozialwissenschaften.

Weichler, K. (2014). Corporate Publishing: Publikationen für Kunden und Multiplikatoren. In A. Zerfass \& M. Piwinger (Hg.), Handbuch Unternehmenskommunikation (S. 767-787). Wiesbaden, DE: Springer Fachmedien.

WEMF AG für Werbemedienforschung (2019). Publikation beglaubigte Auflagezahlen 2019. Abgerufen unter https://wemf.ch/de/kalender/2019/publikation-beglaubigter-auflagezahlen-2019/ (18.03.2019.).

Wessler, H. (2002). Multiple Differenzierung und kommunikative Integration - Symbolische Gemeinschaften und Medien. In K. Imhof, O. Jarren \& R. Blum (Hg.), Integration und Medien (S. 56-76). Wiesbaden, DE: Springer.

Wilson, A., Smith, D., Peel, R., Robertson, J. \& Kypri, K. (2017). A Quantitative Analysis of the Quality and Content of the Health Advice in Popular Australian Magazines. Australian and New Zealand Journal of Public Health, 41(3), 256-258.

Winter, C. (2004), Zeitschrift. In W. Faulstich (Hg.), Grundwissen Medien (S. 454-483). München, DE: Fink. Ytre-Arne, B. (2011). Women's Magazines and the Public Sphere. European Journal of Communication, 26(3), 247-261.

Zangger, C., Glauser, D. \& Becker, R. (2018). The Impact of Modernization and Labor Market Conditions on the School-to-Work Transition in Switzerland: A Dynamic Analysis of the Period from 1946 to 2002. In R. Tillmann, M. Voorpostel \& P. Farago (Hg.), Social Dynamics in Swiss Society (S. 145-159). Cham, $\mathrm{CH}$ : Springer International Publishing. 